\title{
Rough Lattice: A Combination with the Lattice Theory and the Rough Set Theory
}

\author{
Yingchao Shao ${ }^{1, *}$, Li Fu², Fei Hao ${ }^{3}$ and Keyun Qin ${ }^{4}$ \\ ${ }^{1}$ School of Information, Guizhou University of Finance and Economics, Guiyang, Guizhou, 550025, China \\ ${ }^{2}$ School of Mathematics \& Statistics, Qinghai Nationalities University, Xining, Qinghai, 810007, China \\ ${ }^{3}$ Department of Computer Software Engineering, Soonchunhyang University, Asan, 58217,Korea \\ ${ }^{4}$ School of Mathematics Southwest jiaotong university Chengdu, Sichuan, 610031, China \\ ${ }^{*}$ Corresponding author
}

\begin{abstract}
The rough set theory, introduced by Pawlak in 1982, is a formal for dealing with the uncertainties. But it cannot directly deal with the uncertainties with order structure. The lattice theory, introduced by Peirce and Schr\$ddot $\{0\} \$ d e r$ towards the end of the nineteenth century, is a mathematical tool with order structure, algebraic structure and topological structure. In this paper, the rough theory is applied to the lattice theory, and the concept of the rough lattice is presented in order that a tool is presented which can deal with the uncertainties with lattice structure. For this purpose, an equivalence relation on a lattice is defined and then the notions of rough lattice and lower and upper approximations are introduced and some related properties are investigated. At last, some related algebraic structures are studied.
\end{abstract}

Keywords-rough set; lattice; rough lattice; lower approximation; upper approximation

\section{INTRODUCTION}

The theory of rough sets, proposed by Pawlak[20], is a formal tool for modeling and processing incomplete information in information systems. It is an extension of set theory in which a subset of a universe is described by a pair of ordinary sets called lower and upper approximations. By using the concepts of lower and upper approximations, knowledge hidden in information systems may be unraveled and expressed in the form of decision rules. The notion of rough set and its properties have been applied to many areas by some researchers. Some important notions such as rough ring, rough hyperring, rough module and rough group were rough module and rough group were introduced[4,5,6,7,27,28]. In [16], Xiao and Zhang introduced the notions of rough prime ideals and rough fuzzy prime ideals in a semigroup. In[14], Jiang et al. studied the product structures of rough fuzzy sets on a group and proposed the notions of T-rough fuzzy subgroups in a group with respect to a T-fuzzy normal subgroup. Hao et al[12] Searched Minimal Attribute Reduction Sets Based on Combination of the Binary Discernibility Matrix and Graph Theory . In [28],Xiao and Zhang introduced the notions of rough prime ideals and rough fuzzy prime ideals in a semigroup. In [15], Kazanc and Davvaz further introduced the notions of rough prime(primary) ideals and rough fuzzy prime(primary) ideals in a ring and presented some properties of such ideals. Now the rough set theory has been successfully applied to information reduction[3,26,30].
The concept of lattice was introduced by Peirce and Schroder towards the end of the nineteenth century. It derived from pioneering work by Boole on the formalization of propositional logic[2]. In 1930s Birkhoff's work started the general development of lattice theory[11]. Lattice are relatively simple structures since the basic concepts of the theory include only orders, least upper bounds, and greatest lower bounds. Now the lattice theory plays an important role in many disciplines of computer science and engineering. For example, they have applications in distributing, concurrency theory, programming language semantics and data mining. They are also useful in other disciplines of mathematics such as combinatorics, number theory and group theory $[9,1,18]$. Hao and Zhong[13] studied the tag recommendation based on user interest lattice matching.

Presently, the work combining the lattice theory with the other mathematical tools dealing with uncertain information has been initiated. For example, Rana and Roy[24] dealt with rough set approach on lattice theory. Estaji et al.[8] introduced the notion of $\theta$-upper and $\theta$-lower approximations of a fuzzy subset of a lattice. Liu[17]studied generalized rough sets over fuzzy lattices through both the constructive and axiomatic approaches. Estaji et al.[9] studied a connection between rough sets and lattice theory. In this paper, they introduced the concepts of upper and lower rough ideals (filters) in a lattice and then offered some related properties with regard to prime ideals(filters), the set of all fixed points, compact elements and homomorphisms. Some researchers even extended classical rough sets to Boolean algebra[21], completely distributive lattices[3] and residuated lattices[23]. Qin et al.[22]began the study of constructing the lattice structures of soft sets and introduced the concept of soft equality in 2010 and then the study of lattice structure have become one of hot spots in related fields $[10,19,25]$. In this paper we apply the notion of rough sets to the lattice theory and introduce the notion of rough lattice, which is different from that presented by Estaji et.al[24,17,8,9], and then derive some basic properties and discuss the lattice structure of rough ideals (rough filters) of a lattice. The remainder of this paper is organized as follows. Section 2 presents some basic concepts of the lattice theory and the rough set theory. Section 3 presents the notions of rough lattice and derives some related properties. Section 4 concludes the paper. 


\section{PRELIMINARIES}

In this section the subjects needed in the other parts, have been mentioned.

It is well known that a lattice is a partially ordered set in which any two elements $x$ and $y$ have a least upper bound $x \vee y$ and a greatest lower bound $x \wedge y$.

A sublattice $\left(L_{1} ; \vee, \wedge\right)$ of a lattice $(L ; \vee, \wedge)$ is defined on a non-empty subset $L_{1}$ of $L$ with the property that $a, b \in L_{1}$ implies that $a \vee b, \quad a \wedge b \in L_{1} \quad(\vee \vee \wedge \quad$ taken $\left(L_{1} ; \vee, \wedge\right)[2],[11]$.

Next we give some important properties of $\vee$ and $\wedge$.

Proposition1:[11] If $L$ is a lattice, then for all $a, b, c \in L$,

1) $a \vee a=a$ and $a \wedge a=a$;

2) $a \vee b=b \vee a$ and $a \wedge b=b \wedge a$;

3) $a \vee(b \vee c)=(a \vee b) \vee c$ and $a \wedge(b \wedge c)=(a \wedge b) \wedge c$;

4) $a \vee(a \wedge b)=a$ and $a \wedge(a \vee b)=a$.

Proposition 1 presents the characteristic properties of the operations $\vee$ and $\wedge$ in the following theorem.

Theorem1: [11] Let $L$ be a nonempty set equipped with two binary operations $\vee$ and $\wedge$ that satisfy (1)-(4) of Proposition 1. If we define $\leq$ on $L$ by $a \leq b$ if and only if $a \vee b=b$, then $(L ; \leq)$ is a lattice in which the original operations agree with the induced ones, that is, for all $a, b \in L, a \vee b=\sup \{a, b\}$ and $a \wedge b=\inf \{a, b\}$.

Proposition 1 reveals the elegant feature letting lattice be regarded either as ordered sets $(L ; \leq)$ or as algebras $(L ; \vee, \wedge)$.

A nonempty subset $I$ of $L$ is called a lower set of $L$ if for all $x \in L$, there exists $y \in I$ such that $x \leq y$ implies $x \in I$; dually, a nonempty subset $F$ of $L$ is called an upper set of $L$ if for all $x \in L$, there exists $y \in F$ such that $y \leq x$ implies $x \in F$.

\section{Lemma 1[11]:}

1) If $A$ and $B$ are two lower sets of $L$, then so are $A \cap B$ and $A \cup B$.

2) If $A$ and $B$ are two upper sets of $L$, then so are $A \cap B$ and $A \cup B$.

The concepts of ideal and filter of a lattice are usually used and their properties are also usually discussed.

A nonempty subset $I$ of a lattice $L$ is called an ideal of $L$ if it satisfies the follows:
1) $x \vee y \in I$, for all $a, b \in I$;

2) If $x \leq a$,then $x \in I$ for all $a \in I, x \in L$.

Dually, a nonempty subset $F$ of a lattice $L$ is called a filter of $L$ if it satisfies the follows:

1) $x \wedge y \in F$,for all $a, b \in F$;

2) If $a \leq x$,then $x \in F$ fir all $a \in F, x \in L$.

Theorem2[29]: Let $I$ be a nonempty subset of $L$, then the following assertions are equivalent:

1) $I$ is an ideal of $L$;

2) $I$ is a lower set and $I$ is closed under join;

3) $\forall x, y \in L, x \vee y \in I \Leftrightarrow(x, y \in I)$.

dually, let $F$ be a nonempty subset of $L$, then the following assertions are equivalent:

1) $F$ is a filter of $L$;

2) $F$ is an upper set and $F$ is closed under meet;

3) $\forall x, y \in L x \wedge y \in F \Leftrightarrow(x, y \in F)$.

In fact, an ideal $I$ of a lattice $L$ is a lower set of $L$ such that $\forall x, y \in I$ implies $x \vee y \in I$; dually, a filter $F$ of a lattice $L$ is an upper set of $L$ such that $\forall x, y \in L$ implies $x \wedge y \in F$.

The following theorems, which are simple, are used often.

Theorem 3[2,11] Let $I_{1}$ and $I_{2}$ be two ideals of $L$, then so is $I_{1} \cap I_{2}$.

Dually, we have the following theorem:

Theorem 4[2,11]: Let $F_{1}$ and $F_{2}$ be two filter of $L$, then so is $F_{1} \cap F_{2}$.

Definition 1[2]: Let $L$ be a lattice with a least element 0 and a greatest element 1 . An element $a$ is called a complemented element of $L$ if there exists an element $a^{\prime} \in L$ such that $a \wedge a^{\prime}=0$ and $a \vee a^{\prime}=1 . a^{\prime}$ is called a complement of $a$.

Definition 2[2]: An element $a$ of $L$ is called a distributive element of $L$ if $a \vee(x \wedge y)=(a \vee x) \wedge(a \vee y)$, for all $x, y \in L$.

Theorem 5[11]: Let $L$ be a lattice, $a \in L$, then $a$ is a distributive element of $L$ if and only if for all $x, y \in L$, it satisfies the followings,

$$
\text { 1); } a \wedge(x \vee y)=(a \wedge x) \vee(a \wedge y)
$$


2) $a \vee(x \wedge y)=(a \vee x) \wedge(a \vee y)$;

3) $x \wedge(y \vee a)=(x \wedge y) \vee(x \wedge a)$;

4) $x \vee(y \wedge a)=(x \vee y) \wedge(x \vee a)$.

Theorem 6[11]: Let $a$ be a distributive element of $L$, then $a \wedge x=a \wedge y, a \vee x=a \vee y$ implies $x=y$.

An element of $L$ is called a distributive complemented element if it is both a distributive element and a complemented element of $L$. It is clear that any lattice with a least element 0 and a greatest element 1 has at least two distributive complemented elements: 0 and 1 .

In the rest of this section we recall some basic definitions of set approximations.

Let $U$ be a non-empty set of objects called the universe and $R$ an equivalence relation on $U$. We use $[x]_{R}$ to denote an equivalence class in $R$ containing an element $x \in U$. The pair $(U, R)$ is called an approximation space. For any $X \subseteq U$, we can define the lower and upper approximation of $X$ by

$$
R_{-}(X)=\left\{x \mid[X]_{R} \subseteq X\right\}, R^{-}(X)=\left\{x \mid[x]_{R} \cap X \neq \phi\right\},
$$

respectively. The pair $\left(R_{-}(X), R^{-}(X)\right)$ is referred to as the rough set of $X$. The rough set $\left(R_{-}(X), R^{-}(X)\right)$ gives rise to a description of $X$ under the present knowledge, i.e., the classification of $U$. The following proposition is well known and easily seen.

Proposition 2[20]: Let $(U, R)$ be an approximation space. For every subsets $X, Y \subseteq U$, we have

$$
\begin{gathered}
\text { 1) } R_{-}(X) \subseteq X \subseteq R^{-}(X) ; \\
\text { 2) } R^{-}(X \cup Y)=R^{-}(X) \cup R^{-}(Y), \\
R^{-}(X \cap Y) \subseteq R^{-}(X) \cap R^{-}(Y) ;
\end{gathered}
$$

3) $R_{\_}(X) \cup R_{\_}(Y) \subseteq R_{-}(X \cup Y)$, $R_{-}(X \cap Y)=R_{-}(X) \cap R_{-}(Y)$.

By means of the above concepts and operations of rough sets, we can propose the concepts of rough lattices and study some related properties.

\section{ROUGH LATTICES}

Let $L$ be a lattice with a least element 0 and a greatest element 1 and $\varphi$ a mapping $L$ defined as follow: $\varphi(x)=a \wedge x$ for all $x \in L$, where $a$ is a distributive complemented element of $L$. It is trivial to verify that $\varphi$ is a lattice homomorphism.
$R_{a}$ is a binary relation on $L$ by setting: $x R_{a} y$ if and only if $\varphi(x)=\varphi(y)$, that is, $a \wedge x=a \wedge y, \forall x, y \in L$. It is obvious that $R_{a}$ is an equivalence relation on $L$.

Throughout this paper, $L$ is a lattice with a least element 0 and a greatest element $1, a$ is a distributive complemented element of $L, \varphi$ is a mapping on $L$, and $R_{a}$ is an equivalence relation on $L$ as defined in the above unless otherwise specified.

For all $\alpha \in L$, the equivalence class of $\alpha \in L$ is the set $\{x \mid \varphi(x)=\varphi(\alpha)\}$. We denote it by $[\alpha]_{R_{a}}$. By the definitions of upper and lower approximations, we have

$$
R_{a-}(A)=\left\{x \mid[x]_{R_{a}} \subseteq A\right\}, R_{a}^{-}(A)=\left\{x \mid[x]_{R_{a}} \cap A \neq \phi\right\} .
$$

Definition 3: Let $A \subseteq L . A$ is called an upper rough sublattice of $L$ if $R_{a}^{-}(A)$ is a sublattice of $L$. Similarly, $A$ is called a lower rough sublattice of $L$ if $R_{a-}(A)$ is a sublattice of $L$.A subset of $L$ is called a rough sublattice of $L$ if it is both an upper rough sublattice and a lower rough sublattice. For convenience, a rough sublattice of $L$ is called a rough lattice of $L$.Similarly, a subset of $L$ is called a rough ideal of $L$ if its upper approximation and lower approximation are both an ideal of $L$; dually, a subset of $L$ is called a rough filter of $L$ if its upper approximation and lower approximation are both a filter of $L$.

Proposition $3[\alpha]_{R_{a}}$ is a sublattice of $L$ for all $\alpha \in L$.

Proof $\forall x_{1} \in[\alpha]_{R_{a}}, \quad x_{2} \in[\alpha]_{R_{a}} \quad$, we have $\varphi\left(x_{1}\right)=\varphi(\alpha), \varphi\left(x_{2}\right)=\varphi(\alpha)$. Since $\varphi$ is a lattice homomorphism,

$\varphi\left(x_{1} \wedge x_{2}\right)=\varphi\left(x_{1}\right) \wedge \varphi\left(x_{2}\right)=\varphi(\alpha) \wedge \varphi(\alpha)=\varphi(\alpha) \quad$, $\varphi\left(x_{1} \vee x_{2}\right)=\varphi\left(x_{1}\right) \vee \varphi\left(x_{2}\right)=\varphi(\alpha) \vee \varphi(\alpha)=\varphi(\alpha)$,so $x_{1} \wedge x_{2} \in[\alpha]_{R_{a}}, x_{1} \vee x_{2} \in[\alpha]_{R_{a}}$. Thus, $[\alpha]_{R_{a}}$ is a sublattice of $L$ for all $\alpha \in L$.

Suppose that

$$
\begin{gathered}
A \wedge B=\{x \mid x=a \wedge b, a \in A, b \in B\}, \\
A \vee B=\{x \mid x=a \vee b, a \in A, b \in B\} .
\end{gathered}
$$

In general speaking, $A \wedge A=A, A \vee A=A$ don't hold, but we have the following:

Lemma $2 A$ is a sublattice of $L$ if and only if $A \wedge A=A, A \vee A=A$.

Proof: $\Rightarrow$ If $A$ is a sublattice of $L$, then we have $x=x \wedge x$ for all $x \in A$, so $x \in A \wedge A$, therefore, 
$A \subseteq A \wedge A$. On the other hand, for all $x \in A \wedge A$, there exist $a, b \in A$ such that $x=a \wedge b \in A$. Since $A$ is a sublattice of $L$, we can get that $x=a \wedge b \in A$, therefore, $A \wedge A \subseteq A$.Thus, $A \wedge A=A$.

Similarly, we can get that $A \vee A=A$.

$\Leftarrow$ If $A \wedge A=A, A \vee A=A$, then for all $a, b \in A$, we can get that $a \wedge b \in A \wedge A=A$ and $a \vee b \in A \vee A=A$, that is, $a \wedge b \in A, a \vee b \in A$, so $A$ is a sublattice of $L$.

The following lemma helps us to derive some important properties of the rough lattices.

Lemma 3: For all $x \in L, \alpha \in L, \beta \in L$,

1) $[\alpha]_{R_{a}}=[a \wedge \alpha]_{R_{a}}$;

2) $x \in[\alpha]_{R_{a}}$ if and only if $a \wedge x \in[\alpha]_{R_{a}}$.

Proof : 1) It can be get immediately by $\varphi(\alpha)=\varphi(a \wedge \alpha)$.

2) $\Rightarrow$ Since $x \in[\alpha]_{R_{a}}$, we have $a \wedge x=a \wedge \alpha$, so $a \wedge(a \wedge x)=(a \wedge a) \wedge x=a \wedge x=a \wedge \alpha$ ,that is, $a \wedge x \in[\alpha]_{R_{a}}$.

$\Leftarrow$ Since $a \wedge x \in[\alpha]_{R_{a}}$, we have $a \wedge(a \wedge x)=a \wedge \alpha$, so $\quad a \wedge x=(a \wedge a) \wedge x=a \wedge(a \wedge x)=a \wedge \alpha \quad$,that is, $x \in[\alpha]_{R_{a}}$

Proposition 4: For all $\alpha \in L, \beta \in L$,

1) $[\alpha \wedge \beta]_{R_{a}}=[\alpha]_{R_{a}} \wedge[\beta]_{R_{a}}$;

2) $[\alpha \vee \beta]_{R_{a}}=[\alpha]_{R_{a}} \vee[\beta]_{R_{a}}$.

Proof: 1) $\Rightarrow$ For all $x \in[\alpha \wedge \beta]_{R_{a}}$,we have $a \wedge x=a \wedge(\alpha \wedge \beta)$

Let $y=(x \vee(a \wedge \alpha)) \wedge(x \vee(a \wedge \beta))$. On one hand, since $a$ is a a distributive element of $L$, we have $a \wedge y=a \wedge[(x \vee(a \wedge \alpha)) \wedge(x \vee(a \vee \beta))]$

$=[(a \wedge x) \vee(a \wedge \alpha)] \wedge[(a \wedge x) \vee(a \wedge \beta)]$

$=[(a \wedge \alpha \wedge \beta) \vee(a \wedge \alpha)] \wedge[(a \wedge \alpha \wedge \beta) \vee(a \wedge \beta)]$

$=(a \wedge \alpha) \wedge(a \wedge \beta)=a \wedge \alpha \wedge \beta=a \wedge x$, and $a \vee y=a \vee[(x \vee(a \wedge \alpha)) \wedge(x \vee(a \vee \beta))]$

$=[a \vee x \vee(a \wedge \alpha)] \wedge[a \vee x \vee(a \wedge \beta)]$

$=(a \vee x) \wedge(a \vee x)=a \vee x$, so we can get that $x=y$. On

the other hand, since $a \wedge(x \vee(a \wedge \alpha))$ $=(a \wedge x) \vee(a \wedge \alpha) \quad=(a \wedge \alpha \wedge \beta) \vee(a \wedge \alpha)$ $=a \wedge \alpha \in[\alpha]_{R_{a}}$. Thus, we can get that $[\alpha \wedge \beta]_{R_{a}} \subseteq[\alpha]_{R_{a}} \wedge[\beta]_{R_{a}}$. $\quad$ Secondly, for all $x \in[\alpha]_{R_{a}} \wedge[\beta]_{R_{a}}$, we can get that there exist $\alpha_{1} \in[\alpha]_{R_{a}}, \quad \beta_{1} \in[\alpha]_{R_{a}}$ such that $x=\alpha_{1} \wedge \beta_{1}$, so $a \wedge x=a \wedge\left(\alpha_{1} \wedge \beta_{1}\right) \quad=a \wedge \alpha_{1} \wedge a \wedge \beta_{1}$ $=a \wedge \alpha \wedge a \wedge \beta=a \wedge \alpha \wedge \beta$, so $x \in[\alpha \wedge \beta]_{R_{a}}$, that is, $[\alpha]_{R_{a}} \wedge[\beta]_{R_{a}} \subseteq[\alpha \wedge \beta]_{R_{a}}$.

Sum up above, we can get that $[\alpha \wedge \beta]_{R_{a}}=[\alpha]_{R_{a}} \wedge[\beta]_{R_{a}}$.

2) For all $x \in[\alpha \vee \beta]_{R_{a}}$, we have $a \wedge(\alpha \vee \beta)=a \wedge x$. Let

$y=\left[(a \vee x) \vee\left(a^{\prime} \vee \alpha\right)\right] \vee\left[(a \vee x) \vee\left(a^{\prime} \wedge \beta\right)\right]$, where, $a^{\prime}$ is the complement of $a$. On one hand, since $a$ is a distributive complemented element of $L$, we have $a \wedge y=a \wedge\left(\left[(a \vee x) \vee\left(a^{\prime} \vee \alpha\right)\right] \vee\left[(a \vee x) \vee\left(a^{\prime} \wedge \beta\right)\right]\right)$ $=\left(a \wedge\left[(a \vee x) \vee\left(a^{\prime} \vee \alpha\right)\right]\right) \vee\left(a \wedge\left[(a \vee x) \vee\left(a^{\prime} \wedge \beta\right)\right]\right)$ $=\left(a \wedge\left(a^{\prime} \vee \alpha\right)\right) \vee\left(a \wedge\left(a^{\prime} \vee \beta\right)\right)=(a \wedge \alpha) \vee(a \wedge \beta)$ $=a \wedge(\alpha \vee \beta) \quad=a \wedge x \quad$, and $a \vee y=a \vee\left(\left[(a \vee x) \vee\left(a^{\prime} \vee \alpha\right)\right] \vee\left[(a \vee x) \vee\left(a^{\prime} \wedge \beta\right)\right]\right)$ $=\left(a \vee\left[(a \vee x) \vee\left(a^{\prime} \vee \alpha\right)\right]\right) \vee\left(a \vee\left[(a \vee x) \vee\left(a^{\prime} \wedge \beta\right)\right]\right)$ $=\left[(a \vee(a \vee x)) \wedge\left(a \vee\left(a^{\prime} \vee \alpha\right)\right)\right] \vee\left[(a \wedge(a \vee x)) \wedge\left(a \vee\left(a^{\prime} \vee \beta\right)\right)\right]$ $=(a \vee x) \vee(a \vee x)=a \vee x$, So we can get that $x=y$. On the other hand, since $a \wedge\left[(a \vee x) \wedge\left(a^{\prime} \vee \alpha\right)\right]$ $=[a \wedge(a \vee x)] \wedge\left[a \wedge\left(a^{\prime} \vee \alpha\right)\right]$

$=a \wedge\left[\left(a \wedge a^{\prime}\right) \vee(a \wedge \alpha)\right] \quad=a \wedge \alpha \in[\alpha]_{R_{a}} \quad$,and $a \wedge\left[(a \vee x) \wedge\left(a^{\prime} \vee \beta\right)\right]=[a \wedge(a \vee x)] \wedge\left[a \wedge\left(a^{\prime} \vee \beta\right)\right]$ $=a \wedge\left[\left(a \wedge a^{\prime}\right) \vee(a \wedge \beta)\right]=a \wedge \beta \in[\beta]_{R_{a}} \quad$,so $\quad$ we have $y \in[\alpha]_{R_{a}} \vee[\beta]_{R_{a}}$, which implies $x \in[\alpha]_{R_{a}} \vee[\beta]_{R_{a}}$.

Thus, we can get that $[\alpha \vee \beta]_{R_{a}} \subseteq[\alpha]_{R_{a}} \vee[\beta]_{R_{a}}$. Secondly, for all $x \in[\alpha]_{R_{a}} \vee[\beta]_{R_{a}}$, there exist $\alpha_{1} \in[\alpha]_{R_{a}}$, $\beta_{1} \in[\alpha]_{R_{a}}$ such that $a \wedge \alpha=a \wedge \alpha_{1}, a \wedge \beta=a \wedge \beta_{1}$ and $x=\alpha_{1} \vee \beta_{1} \quad$ so $\quad a \wedge x=a \wedge\left(\alpha_{1} \vee \beta_{1}\right)$ $=\left(a \wedge \alpha_{1}\right) \vee\left(a \wedge \beta_{1}\right)$

$=(a \wedge \alpha) \vee(a \wedge \beta) \quad=a \wedge(\alpha \vee \beta)$, that is, $x \in[\alpha \vee \beta]_{R_{a}}$. Thus, we have $[\alpha]_{R_{a}} \vee[\beta]_{R_{a}} \subseteq[\alpha \vee \beta]_{R_{a}}$.

Sum up above, we can get that $[\alpha \vee \beta]_{R_{a}}=[\alpha]_{R_{a}} \vee[\beta]_{R_{a}}$. 
By the proof of Proposition 4, we can get that Proposition $4(1)$ is still true even if $a$ is not a complemented element of $L$.

\section{CONCLUSION}

We have proposed the concepts of rough lattices and studied their basic properties. Although many results reported here are only concerned with basic properties about these new notions, they can hopefully provide more insight into and a full understanding of rough sets theory and one could see that this study presents a very preliminary, but potentially interesting research direction. Naturally, a very important and interesting problem is how to apply the models given in this paper to build the corresponding theory of knowledge discovery in lattice-valued information systems.

\section{ACKNOWLEDGMENT}

The authors are indebted to Professor F. Feng for helpful discussion and valuable suggestions.

This work was supported by Qinghai Science Foundation(Grant No.2013-Z-913) and Science and Technology Fund Project of Guizhou Province(Grant No.LKB[2012]02) .

\section{REFERENCES}

[1] N.Ajmal and A.Jain, "Some constructions of the join of fuzzy subgroups and certain lattices of fuzzy subgroups with supproperty", Information Sciences,vol,179,pp.4070-4082,1995

[2] G.Birkhoff, lemph\{Lattice Theory\}, Trans. Amer. Math. Soc., New York, 1967

[3] D.Chen, W.Zhang, D.Yeung and E.C.C. Tsang, "Rough approximations on a complete completely distributive lattice with applications to generalized rough sets", Information Sciences,vol,176, pp.18291848,2006.

[4] B.Davvaz, "Roughness in rings", Information Sciences, vol, 164, pp.147-163,2004

[5] B.Davvaz, "Roughness based on fuzzy ideals",Information Sciences,vol, 176, pp.2417-2437,2006

[6] B.Davvaz, “Approximations in hyperrings”, Journal of MultipleValued Logic and Soft computing 15(2009)471-488

[7] B.Davvaz, M.Mahdavipour, "Roughness in modules", Information Sciences ,vol, 176, pp. 3658-3674,2006

[8] A.A.Estaji, S.Khodaii and S.Bahrami, "On rough set and fuzzy sublattice”, Information Sciences,vol, 181,pp.3981-3994,2011

[9] A.A.Estaji, M.R.Hooshmandasl and B.Davvaz, "Rough set theory applied to lattice theory”, Information Sciences, vol,200 ,pp.108122,2012

[10] L. Fu, “Soft Lattices”, Global Journal of Science Frontier Research. Vol,10(4), pp.157-159,2010

[11] G.Gratzer, “General Lattice Theory”, Academic Press, INC. 1978

[12] F.Hao, Z.Pei and S.Zhong, "Searching Minimal Attribute Reduction Sets Based on Combination of the Binary Discernibility Matrix and Graph Theory”, International Conference on Fuzzy Systems (FUZZIEEE 2008), pp.54-57

[13] F.Hao and S.Zhong, "Tag Recommendation Based on User Interest Lattice Matching”, International Conference on Computer Science and Information Technology (ICCSIT2010), pp.276-280

[14] J.Jiang, C.Wu and D. Chen, "The product structure of fuzzy rough sets on a group and the rough T-fuzzy Group”, Information Science, vol,175, pp.97-107,2005
[15] O.Kazanc and B.Davvaz, "On the structure of rough prime(primary) ideals and rough fuzzy prime(primary)ideals in commutative ring”, Information Sciences ,vol,178,pp.1343-1354,2008

[16] [N.Kuroki and P.P.Wang, "The lower and upper approximations in a fuzzy Group”, Information Sciences, vol,90,pp.203-220,1996

[17] G.Liu, "Generalized rough sets over fuzzy lattices”, Information Sciences , vol,178,pp.1651-1662,2008

[18] Y.Li, "Finite automata theory with membership values in lattices", Inform. Sci., vol,181(5),pp.1003-1017,2011

[19] M.Marudai and V.Rajendran, "New Construction of Fuzzy Soft Lattices”,International Journal of Computer Applications, vol,23(1), pp.33-38,2011

[20] Z. Pawlak, "Rough sets", International Journal of Computer and Information Sciences ,vol,11,pp.341-356,1982

[21] G.Qi and W.Liu, "Rough operations on Boolean algebras", Information Sciences,vol,173,pp.49-63,2010

[22] K.Qin and Z.Hong, “ On soft equality”, J.Comput.Appl.Math. Vol,34,pp.1347-1355,2010

[23] A.M.Radzikowska and E.E.Kerre, "Fuzzy rough sets based on residuated lattices”, Transactions on Rough Sets, Lecture Notes in Computer Sciences, vol,3135,pp.278-296,2004

[24] D.Rana and S.K.Roy, "Rough Set Approach on Lattice”, Journal of Uncertain Systems, vol,5(1),pp.72-80,2011

[25] Y.Shao and K.Qin, "Fuzzy Soft Sets and Fuzzy Soft Lattices", International Journal of Computational Intelligence Systems, vol,5(6),pp. 1135-1147,2012

[26] D.Slezak and W.Ziarko, "The investigation of the Bayesian rough set Model”, International Journal of Approximate Reasoning, vol, 40,pp.81-91,2005

[27] C.Wang and D.Chen, "A short note on some properties of rough groups", Computers and Mathematics with Applications, vol, 59,pp.431-436,2010

[28] Q.Xiao and Z.Zhang, "Rough prime ideals and rough fuzzy prime ideals in Semigroups”, Information Sciences ,vol,176,pp.725-733,2006

[29] X. Zhang, "Fuzzy logic and its algebraic analysis”, Science Press. 2008.(In Chinese).

[30] W.Zhu and F.Wang, "Reduction and axiomization of covering generalized rough Sets”, Information Sciences vol,152,pp. 217230,2003 